\title{
Wearable Performance
}

\author{
Johannes Birringer \& Michèle Danjoux \\ DAP-Lab, Brunel University, West London \\ johannes.birringer@brunel.ac.uk; michele.danjoux@ntu.ac.uk
}

\begin{abstract}
Wearable computing devices worn on the body provide the potential for digital interaction in the world. A new stage of computing technology at the beginning of the 21 st Century links the personal and the pervasive through mobile wearables. The convergence between the miniaturisation of microchips (nanotechnology), intelligent textile or interfacial materials production, advances in biotechnology and the growth of wireless, ubiquitous computing emphasizes not only mobility but integration into clothing or the human body. In artistic contexts one expects such integrated wearable devices to have the two-way function of interface instruments (e.g. sensor data acquisition and exchange) worn for particular purposes, either for communication with the environment or various aesthetic and compositional expressions. "Wearable Performance" briefly surveys the context for wearables in the performance arts and distinguishes display and performative/interfacial garments. It then focuses on the authors' experiments with "design in motion" and digital performance, examining prototyping at the DAP-Lab which involves transdisciplinary convergences between fashion and dance, interactive system architecture, electronic textiles, wearable technologies, and digital animation. The concept of an "evolving" garment design that is materialized (mobilized) in live performance between partners originates from DAP Lab's work with telepresence and distributed media addressing the "connective tissues" and "wearabilities" of projected bodies through a study of shared embodiment and perception/proprioception in the wearer (tactile sensory processing). Such notions of wearability are applied both to the immediate sensory processing on the performer's body and to the processing of the responsive, animate environment.
\end{abstract}

Keywords: digital performance, design in motion, wearable space, emotional design, post-choreography, narrative 


\section{Performance and fashion}

The Czech dance journal Tanecni zóna published a special issue in 2006 titled "Dance and Fashion" which looked back at Prague Fashion Week to examine the particular styles of presentation chosen by designers to emphasize concepts and themes based in performance theory, anthropology and "cultural sciences" that use fashion behavior to interpret psychological or sociological issues. The July 2008 issue of $i$ - $D$ magazine featured the "visionary" fashion designers Viktor and Rolf and their collaboration with makeup company Shu Uemura on a limited triptych of false eyelashes (Hanra 2008), alongside American theatre designer/director Robert Wilson, invited by NIKE to "to create a series of high-definition portraits" of athletes to coincide with the launch of NIKE's new collection "Nike Sportswear" in conjunction with the 2008 Beijing Olympics (Jones 2008). Yohji Yamamoto is reported to stay "one step ahead of the game by constantly taking fashion to places you would not expect to see it." The magazine mentions his one-off collection produced for a Chinese cultural organization and his short concept for the film project "Four cities in motion and a human story of conflict," directed by photographer Max Vadukul. Choreographed by Rachel Lopez de la Nieta and Ben Hash, the film, promoting Yamamoto's "Coming Soon" collection, features dancers moving dynamically through the city. Fabric, skin, air and movement are the focus, with Vadukul explaining that " $80 \%$ of expression is within movement of its negative and positive space" (Cochrane 2008).

Our paper will introduce the research project "Design in Motion," connecting fashion and performance through a conceptual approach that locates expression within and through movement and is concerned with dynamic methods of co-creation and sensortized design. Although fashion design has not yet played a strong role in the research sectors of digital arts, technology and science, its strategic links with materials science in the current era of smart fabrics and wearable technologies are now recognized, as well as its historical associations with the moving image, photography and animation. The London Science Museum/Dana Centre, for example, recently hosted the symposium "The Fashion for Smart Materials" (September 2008) to introduce some of the innovative materials technologies challenges for fashion and 
design, drawing attention to the experimental design/science labs created in the UK to support longer-term research partnerships.

While our choreographic design method extends the working processes and conventional methods of static 2-D/3-D design development generally used by fashion designers, "Design in Motion" also seeks to re-contextualize fashion through such collaborative and interdisciplinary working practices as they were suggested by the "Fashion for Smart Materials" consortium (SMART.mat), deriving ideas from film, architecture, digital media art, mobile communications and interactive performance. Online collaboration and telepresence were also explored throughout the stages of the research process (2004-2007). Our artistic work positions fashion as interfacial medium and narrative tool, looking beyond pure surface display at a more deeply searching communication - and dialogue - of inside to outside. It aims to challenge the boundaries of what might be possible technically, aesthetically and creatively and searches for a new paradigm for fashion.

Fashion has indeed always been in the business of collaboration; with film (e.g. Coco Chanel, Jean Paul Gaultier), art and architecture (cf. Skin+Bones: Parallel Practices in Fashion and Architecture at London's Somerset House, 2008, featuring over 50 internationally-renowned architects and designers including Alexander McQueen, Vivienne Westwood, Comme des Garcons, Yohji Yamamoto, Future Systems, Frank Gehry, and Zaha Hadid's "fashion" buildings and "construct" garments), and live art installations of the kind that Vanessa Beecroft or Christian Rizzo have staged with models or dancers. In a review of Angelin Preljocaj's new ballet Blanche Neige, dance critic Thomas Hahn emphasizes that Jean Paul Gaultier's costumes continue a long tradition of collaboration between designers and choreographers, e.g. Coco Chanel designing for the Ballet Russes, Issey Miyake for William Forsythe, Rei Kawakubo for Merce Cunningham, Narciso Rodriguez for Christopher Wheeldon, Dries van Noten for Anne Teresa de Keersmaeker, Giorgio Armani for John Neumeier, or Gianni Versace for Maurice Béjart (Hahn 2009).

Interdisciplinary practices in fashion have recently brought us the biodegradable dresses of Helen Storey and Tony Ryan or the fermented "Micro'be"" garments 
synthesized by Donna Franklin at the Symbiotica Lab in Perth where fashion fuses with science, and the visually communicating dresses of designers experimenting with wearable technologies, such as Joey Berzowska, Barbara Layne, CuteCircuit, Viktor and Rolf, and Hussein Chalayan. Chalayan's exhibition "From fashion and back" is currently on view at the Design Museum in London (January 22 - May 17, 2009) and demonstrates some of the significant cross-overs between fashion concepts and art, as well as fashion design's increasing acceptance within fine arts contexts of display. Earlier "costumed" audio sculptures had been displayed in performance by the French director and sound artist Benoît Maubrey whose "Audio Ballerinas" - wearing specially designed amplified dresses - performed at festivals or on the streets in many parts of the world.

\section{Wearables in Design and Performance}

The historical lineage of such performed wearable designs can be traced back much further, for example to Oskar Schlemmer's Triadic Ballet or Metal Dance created at the Bauhaus in the 1920s, or Loïe Fuller's “electric dresses" used in her dance experimentations with special lighting and color at the beginning of the $20^{\text {th }}$ Century, right through to Brazilian artist Hélio Oiticica's parangolés - colorful layered garments worn by Samba dancers and visitors to his environmental exhibitions in the 1960s, and to U.S. performance artist Laurie Anderson's use of her own amplified body as a percussion instrument in concerts. The relationship of fashion and wearable computing to music - and especially to computer music's understanding of the body in interactive, gesture-controlled performance as a kind of "extended instrument" awaits further exploration. Singer/digital artist Julie Bokowiec, for instance, focuses on gestural techniques and "kinaesonics" (Bokowiec and Bokowiec 2006), while others emphasize the potential of "intimate computing" for the tactile, kinaesthetic and affective dimensions of interaction design (Hansen and Kozel 2007).

Short lead times and quick turn overs in fashion construction (for the seasonal collections) often prevent designers in the creative industries to dwell on more sustained research into new materials and the mobility or transformability afforded by wearable technology. Performative or aesthetic values also differ from the more functional (high performance) values demanded from clothes and accessories used in 
the sports, health, military and safety sectors. Current interdisciplinary advances in the meeting of smart materials and wearable design have resulted in initiatives such as the Smart Clothes and Wearable Technology Research Group (Newport, Wales) and MADE (Materials and Design Exchange) in the UK or the Interactive Textiles and Wearable Computer Group at Hexagram (Montréal, Canada). They focus on knowledge transfer and workshops, support innovation (such as the planned creation of a Science Fashion Lab in Cambridge), and help drive the new "sensory design" field interested in smart clothing that addresses so-called end-user needs from technical, aesthetic and cultural viewpoints. It is an emerging field looking for convergences (for example with Nokia's research into haptics, vibrotactile feedback and flexible/modular surfaces) and a future of sensing/communicating clothing that can express people's personalities, needs and desires, or augment social dynamics through the use of wearables as "theatre" and as emotional "tools." Such expressive and emotional design factors were discussed at length, for instance, during the "Design and Emotion" conference at Chalmers University of Technology in Gothenburg (2006), where designers of industrial products and accessories (jewellery, flexible LED displays, etc) dwelled on the affective messages conveyed through kansei features of their applications. The Japanese notion of kansei (emotion design) implies a stronger emphasis on the wearable as a social medium, capturing user subjectivity and intimacy. While the latter purpose is of great interest for performers, the artistic experimentation with smart technologies is of course not market-oriented and has no end-user. Rather, the mobilization of smart technology concerns sensorial experience and expression (involving transformations of the performer-audience relations), a more experimental and playful adaptation of the digital medium as a wearable medium.

At this point we can distinguish two categories of wearables used performatively: (1) Intelligent garments such as Joanna Berzowska's "Memory Rich Clothing" prototypes, which record intimate actions felt on the clothes; or Barbara Layne's reactive "Jacket Antics," outerwear that responds to surface contact or built in programming. Layne's garments are woven with light emitting diodes (LED's), microcontrollers and sensors that can generate a scrolling message when the wearers hold hands; the fabric itself is the circuit board and completely flexible as it is woven 
into the structure. Kerri Wallace's thermochromic shirts react to body temperature and are inspired by ideas of color mutation and biomimetics. Thermochromic, electroluminescent, scent-emitting, and transformational e-broidery garments, or Chalayan's shape-shifting, mechanically morphing "Flip Through" dresses (Spring 2007) and "Video Dress" (Fall 2007) prototypes also belong to this category as the electronic, chemical or mechanical effects occur within the worn materials themselves (Birringer 2008, pp. 214-32; Seymour 2008). Such surface effects have a display character that lends itself to theatrical and intimate dialog, but they don't seem to demand particular performance techniques. Similarly, Jane Harris's work explores the portrayal of textile motion in the realm of 3D computer graphic visualization; her digital animations (Potential Beauty), first exhibited in the UK in 2002-2003, focused on the dreamlike movement of the dresses alone, insofar as the actual wearer of the garments was deleted in the final screen version. Tina Gonsalves's Medulla Intimata (2002-2004) are video displays of wearables - often deploying obtrusive technologies to monitor the body - that probed the wearer's emotional feelings. Her current work continues this research into "emotion sensing"; she collaborates with affective computing scientists, human computer interaction specialists and neuroscientists to develop more transparent monitoring techniques. None of the prototypes mentioned above have been used in a performing arts context.

(2) Interfacial garments sensing and "actuating" the virtual environment through sensorial embodiment offer greater potential for a relational aesthetics, as performers can affect changes in an audio-visual digital environment or even seek to integrate audience behavior into the reactive functions of the environment, as it was demonstrated by Penelope Wehrli's camera orfeo and kondition pluriel's passage, two choreographic installations exhibited at the CYNETart 2008 festival in Dresden/Hellerau (Birringer 2008b). Such "wearing” of the space, as we call it, produces transformations in the behavior of responsive environments, and the careful modulation of such interrelations with a reciprocating context requires a performer technique. It at least requires a familiarity with the dimensions of real-time composition implied by such transductions. Music, theatre, dance, and locative media performances have experimented with presenting transductive embodiment. Installation art (e.g. Thecla Schiphorst's soft( $n$ ) and exhale: breath between bodies) in 
particular probes a wide range of affective interfaces for users but only very rarely "gives away" intelligent garments to be worn by participating audiences (Schiphorst 2006). Artistic contexts in many cases privilege the trained performer to articulate the wearable as a medium of representation.

\section{Design in motion}

In our own work, the collaboration between choreographer, designer, digital artists, composers and engineers focuses on the real-time relationship between the tactile performance experience of the garments and the projective visual and acoustic/sonic media environment. In the design phases for our production of Suna no Onna, first presented at London's Laban Theatre in December 2007, the animation of the garments and the cloth took centre stage as the dancers found particular expressive articulations of dramatic character through the kinesthetic, tactile and material experience. The kinesthetic and gestural movement was echoed in the projected digital environments - the moving landscapes and graphic details of the world of the characters. We also anticipated that the visual and tactile dimensions of performance have significant acoustic implications, that color, texture and light have tonal resonances, and that some of the garment materials themselves generate sound and invite being amplified, sampled and remanipulated through sound software.

Initially, the convergence of art forms, scientific research, and network capabilities provoked our thinking about design processes as connective audiovisual resonances. We imagined garments to be extruded into telepresence, the "tissues" of screen projection, while also noticing the many possibilities of capture technologies to retrace corporeal and sartorial images, converting the dynamics of moving design through algorithmic mappings of the kinetic data into a virtual format where data can be varied infinitely and transmitted between geographically distant wearers. We had worked with telematic partners in Japan, USA and Italy at the time, and our telepresence performances taught us to give attention to small gestures, slow constructions (keeping one's balance in motion during the coiling and folding/unfolding of cloth on and around the body), and subtle details in "drawing" out the movement of the garments and their visualization on the screens. (Fig.1) 


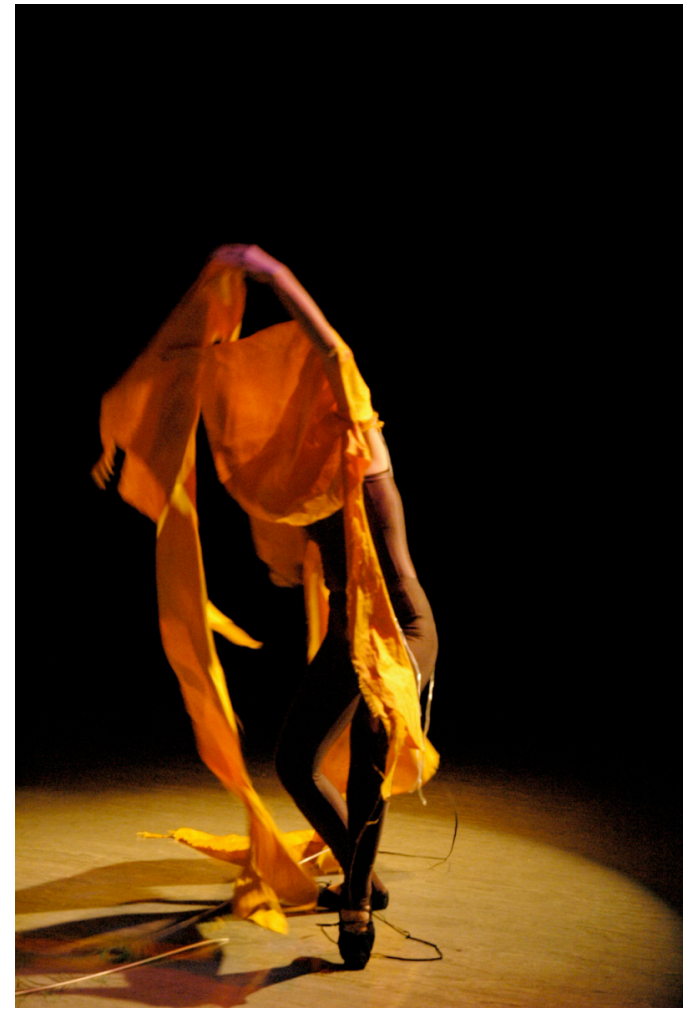

Fig. 1 Helenna Ren manipulating her partial garment in performance. Videostill ( 2005 J. Birringer

Motion capture and video projection, 3-D animation and moving graphics augment the performance reality. Our starting point for the "Emergent Dress" project, however, was the performer's movement improvisation, adjusting and readjusting the worn garments (in partial design states) through a careful interaction with the participating screen space, creating a dialog across the network with other, remote performers. Or, rather, with the projected (streaming) images of remote performancegarments. Like architects looking at constructible forms for edges and joints, for the points where surfaces or different materials meet, our team composed garment materials into animated images through performance with camera tracking and microphones. Tedr (Telematic Dress), first performed in 2005, was directed at the intimacy of the wearable experience, the desire and erotic sensuality attached to the clothes worn on the skin, the frivolous, extroverted but also secretive (even antiaesthetic, camouflaging) dimensions of fashioning appearance, and the physiological processes and patterns through which the proprioceptive systems attend to the body's wearing of itself. This emphasis on intimacy within the material-informational composition - a fluid boundary of continuous real-time construction and 
reconstruction - evolved from dancer Helenna Ren's telepresence interaction with remote partners. She tested all of the versions of the transformable body-garment and the digitally manipulated garment-body - prototypes we named "ScreenDress" and "SensorDress." In the development of the prototypes, the corset became our first vehicle - conceptual metaphor and material object - along with other parts and fragments of pink, yellow and black cloth that Ren put on, assembled and disassembled in her dance of wearing, gesturing, folding, stretching, unbinding fabrics and needles, moving and re-moving gloves, shifting elements of the garments into and out of focus, while interacting with the physical camera (moved in close proximity by the camera operator). (Fig.2)

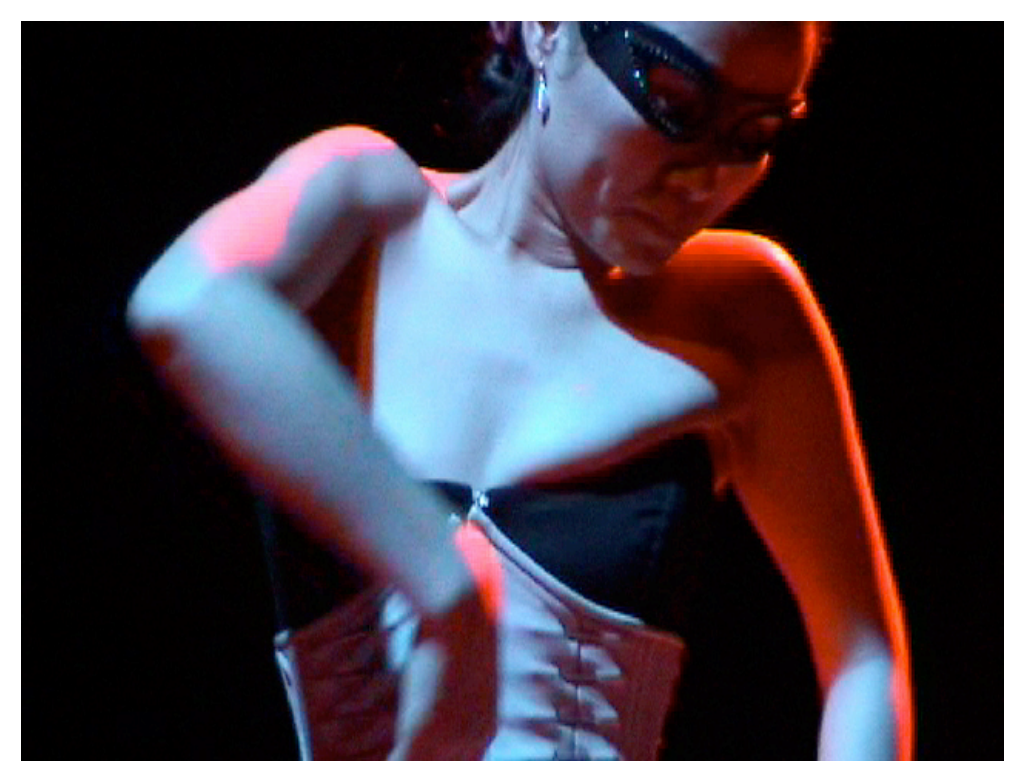

Fig. 2 Helenna Ren dancing in Klüver, a film featuring the corsetbody concept designed by Michèle Danjoux. Videostill (C) 2005 J. Birringer

\section{ScreenDress}

The first version of ScreenDress was a composite virtual fashion performance. In dialogues enacted in real-time with remote performers Natalie King, Keira Hart and Joe Willie Smith, characters (“Zorro", "Houdini”, and "Klüver”) emerged gradually as the dancer began to shape the information composed with the corsetbody. The video images of Ren's movement with the garment were sent to our Arizona partners' screen environment (constructed as a garden of large hanging leaves) and projected onto the leaves as well as a luscious white Victorian dress suspended from the ceiling, 
functioning as the centre-piece of our partners' experiment with haptic memory. Helen Raleigh, AJ Niehaus and Galina Mihaleva devised the conceptual strategies with which the participants in Arizona used this memory-rich garment as a sensory sculpture and smart surface. Their dancers could step inside the surface and wear it, shedding their other clothes and embedding themselves inside an older (historical) garment. They inserted themselves into a kind of "habitat" which enveloped them.

Telematic performance challenges the dualism of literal and virtual garment. We experienced the suspended dress as a receptacle, a sensory surface which functions as a mnemonic landscape and an instrument responding to touch, its built-in sensors generating a sonic text (words recorded in Joe Willie Smith's voice). The "Victorian" dress flared into temporary focus, historical images or fragmented film stills from a natural landscape appearing on it, then all discrete traces disappeared again, and as Ren's telepresent corsetbody remobilized it, distance and proximity became interwoven, viewable and feelable from the inside out. (Fig.3) When we speak of inside out, we think of the dancer's intensions vis à vis the telepresent projections which could be considered a kind of mask: wearing the mask necessitates motivated attention and intensity of focus on part of the performer in order to experience the digital as a direct extension of the felt, phenomenological body with its sensorimotor and neural systems.
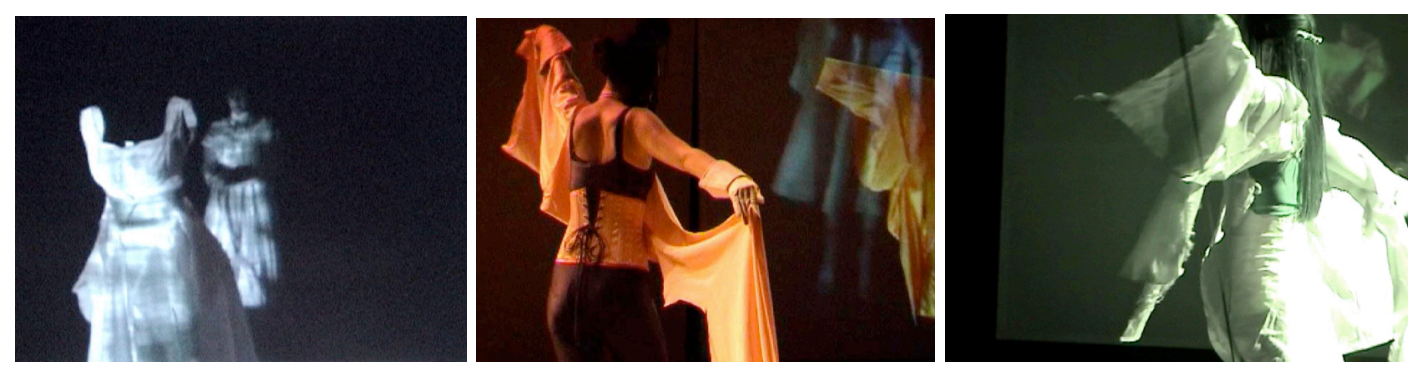

Fig. 3 Keira Hart behind Victorian dress (left), Helenna Ren in front of Victorian dress (middle), and merging her movement with it (right). Videostills (C) 2005 J.Birringer

\section{SensorDress}

This stimulating, porous quality of the digital garments, which feed information back into the dancing body, inspired us to develop the temporal design concepts for the wearable space in Suna no Onna and our ongoing research into emotionally narrative composition. Our interest in adapting a film (Hiroshi Teshigahara's 1964 cinematic 
interpretation of Kobo Abe's novel Woman in the Dunes) to a real-time processing performance arose from our observations of Helenna Ren's and Katsura Isobe's rehearsals with garments that can evoke an almost existential state or metabolism. Here one might have to take recourse to subjective perception, of course, in order to translate patterns of activation we imagine or feel or associate as resulting from experiences of sensorial gesture, the touching of cloth and the bearing of the weight (or lightness) of garments, the textural distinctness of materials on skin, the glove on the hand, the vibrations of color, the rustle of the folds. There is the magic of light falling on matter, the refractions of color and image surround us on the projected graphic landscapes - the real and surreal video still sequences, digital animations and their calligraphic momentum, surfaces seemingly elastic or energized, shimmering fields of windblown sand, strange insects and spirits crawling out of the dunes.

Consequently, we began to think of the digitally projected dunes landscape as an extended mantle in itself, an environment that dresses the performer and, at the same time, is actuated by the performer's sensortized garments. (Fig.4)

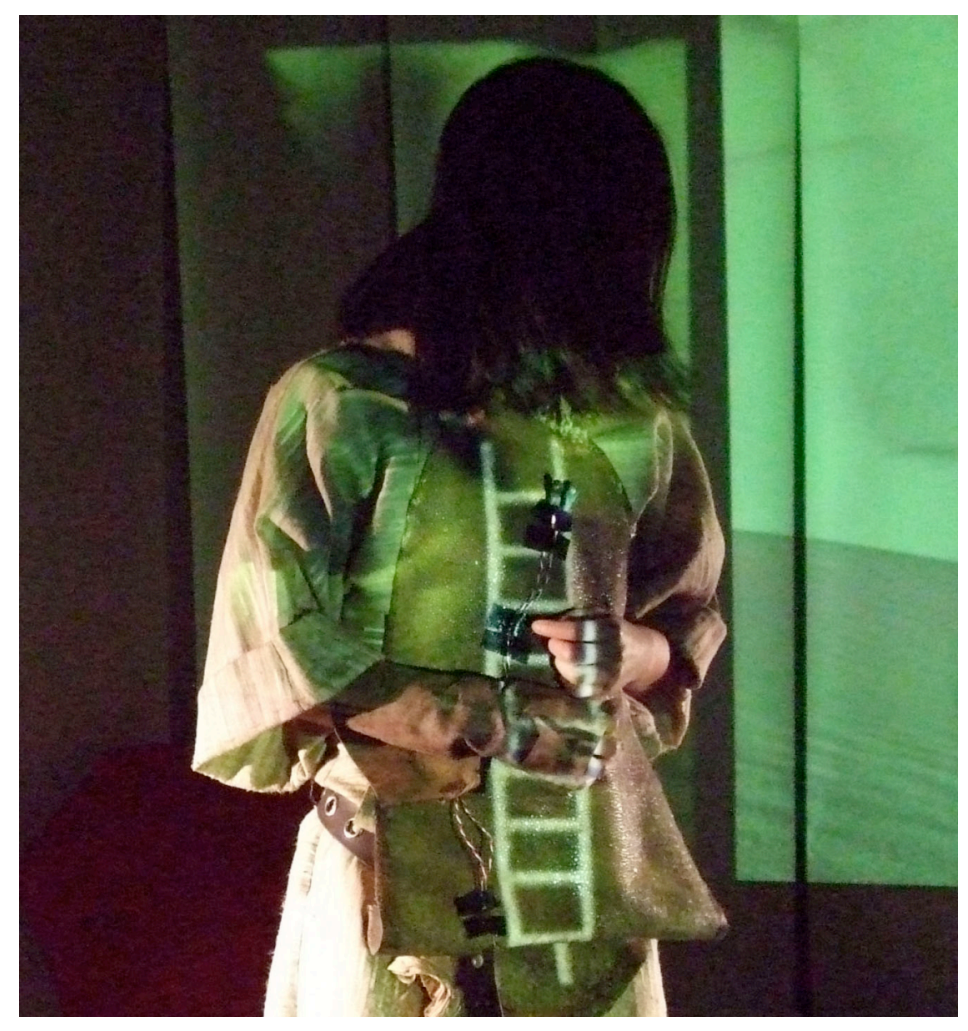

Fig. 4 Interfacial design development: first SensorDress prototype for Katsura Isobe's role of woman in the dunes (Suna no Onna), with mapping of technologies (photocell sensors) and projection onto fish skin. Photo (C) 2007 Michèle Danjoux 


\title{
6 The Teshigahara collection and wearable space
}

Porousness and motion were key ideas we found in the original novel, The Woman in the Dunes, when Abe writes that

\begin{abstract}
Certainly sand was not suitable for life. Yet, was a stationary condition absolutely indispensable for existence? Didn't unpleasant competition arise precisely because one tried to cling to a fixed position? If one were give up a fixed position and abandon oneself to the movement of the sands, competition would soon stop. Actually, in the deserts flowers bloomed and insects and other animals lived their lives. These creatures were able to escape competition through their great ability to adjust... While he mused on the effect of the flowing sands, he was seized from time to time by hallucinations in which he himself began to move with the flow (Abe 2006, p.15).
\end{abstract}

Inspired by Hiroshi Teshigahara's intense black and white film, our exhibition of Suna no Onna is a slow moving sensual dance, a wordless drama merging virtual and real images of a life of existential entrapment in an apparently inhospitable und unstable habitat. The ominous sand dunes of Teshigahara's desert are transformed into a virtual projected environment that shapes the unconscious ground where the Woman (Isobe) meets a scientist-foreigner (Olu Taiwo) who stumbles into her life to become her captive. His captivity turns into a process of adaptation to the environment and a modification of the idea of escape. A third composite character (BlueSpirit, FolkWoman, Insect, EvilVillage) is created by Ren who at times interacts with virtual screen phantasms whose digital movement animation is created from motion-captured data and, to a certain extent, resonates with Japanese anime (for example the extraordinary Baburugamu kuraishisu [Katsuhito Akiyama et al, 1987]). The immersive projection environment (suspended paper rolls forming a threedimensional curved space) envelops the mid- and downstage regions where the dancers portray their characters in an eclectic mix of Japanese, Chinese and African performance vocabularies. 
The character studies and elliptical narratives adapted from the film formed the basis of constructing the digital movement composition for Suna no Onna. But the movement also arose from the particular garments, their temporal and modular forms. Designing the collection followed an inverse pattern; a design process conventionally begins with the 2-D design sketch and the static form. From here ideas are developed two and three-dimensionally in preparation for final 3-D garment realization. Yet, our "Emergent Dress" project didn't start with the design sketch or static state. Instead we explored movement, introducing partial garment structures and cloth to the initial frame: inviting the dancer to move with cloth, sensing and experiencing the qualities of the cloth (in front of our eyes/the camera eye), its potential and design possibilities. We analyzed the movement reactions initiated by the tactile stimulus of the cloth and considered how garment form and structure emerge temporally, i.e. through synergies of performance, sound, lighting and movement-image (on the projection screens).(Fig.5)

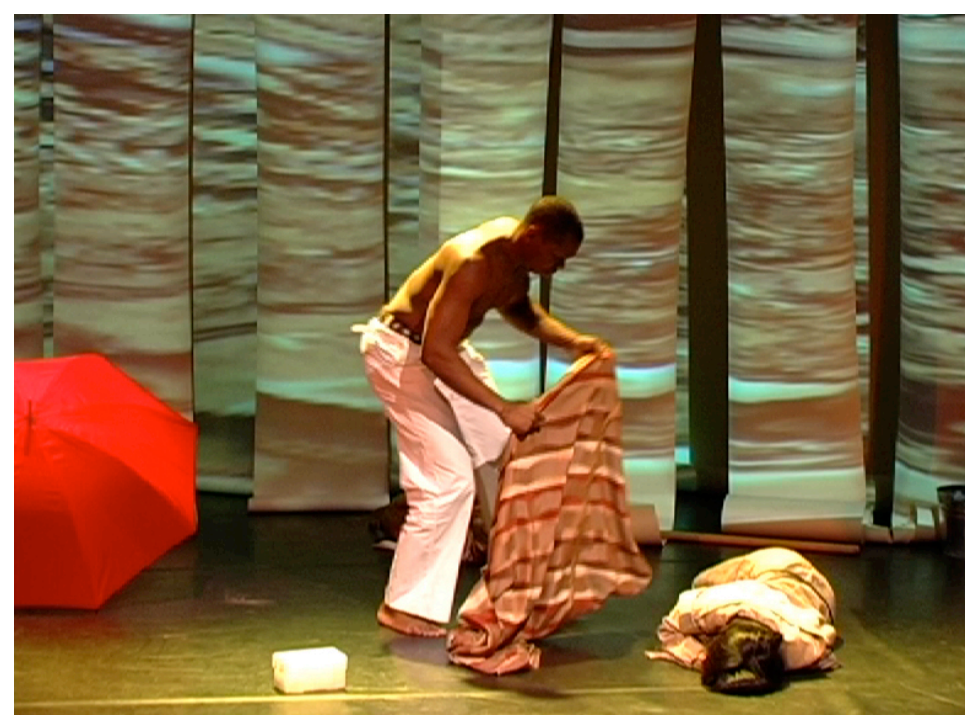

Fig. 5 Olu Taiwo with Katsura Isobe as the duneswoman, wearing rhythmic textures of the movement sensitive SandCoat. Photo (C) 2007 Michèle Danjoux

The choreography of garment conjoins the interactive choreography of the body with the responsive environment. Clothing has its effects on the body. It moves the body into a specific pose and its expression, while the integrated sensors provide the motion sensing data for the activation of the 3-D digital projection environment. This approach to the generation of the "wearable space" within the real-time processing scenario means that the moving body has an impact on how the form is fashioned and 
the fashioned form impacts on the sensing moving body and how it affects the virtual space. The corporeal experience is divergent from the projected 3-D environment, since the performer does not navigate inside immersive VR (which would require head-tracking or data glove interfaces), yet the unrestricted full bodied movement allows a continuous sensation of proprioceptive virtuality as the image presences move with the dancer in close, luminous contact (cf. Munster 2006, pp. 101-16).

Furthermore, there are scenes in which the performers are also onscreen and where the seeming divergence collapses into a mutual sharing of space. The second version of the ScreenDress is constructed from Chromatte, a technical light-reflecting cloth for chroma key production in TV and film. This material, designed to work dynamically with a LiteRing (a camera mounted device featuring LED's) utilizes the retro-reflective properties of its fabrication for live effects/image replacement, fusing motion graphics with onscreen performance. The physical form of the garment, existing in the real world, is gunmetal grey which turns green when reflecting the camera LiteRing. On the material level, we are concerned with the cut and the fabrication, the detailing and finishes, and the overall silhouette statement, the structure of the garment and how the body engages with the piece, i.e. how it is worn/performed and choreographed into movement. On the iconic level, the wearable is transposed to the screen where it morphs into the graphic images edited into it. The dress now displays animated "time-images" and "crystal-images" (Deleuze 1989), it becomes a moving screen within the screen. In Deleuze's theory of film and the projected image, a threshold or an "outside" is carried into the interior of film through editing cuts and intervals that disrupt narrative continuity or logical causality: the image movement succumbs to ambiguous temporalities as well as irrational or artificial spatialities (and anime-like "decorative" surfaces). The Deleuzian "method of between" or interval (Deleuze 1989, p. 180) also comes into play in our scenography through the multiply split and irregularly suspended paper scrolls which create spatialized intervals on the projected stage that can be inhabited by the performers and, in extension, the viewers. The viewers experience this crystallization of time somatically and kinaesthetically, as they perceive the dancers" "wearing" and intensification of sound and images through the slow or sudden displacements of the real. (Fig. 6) 


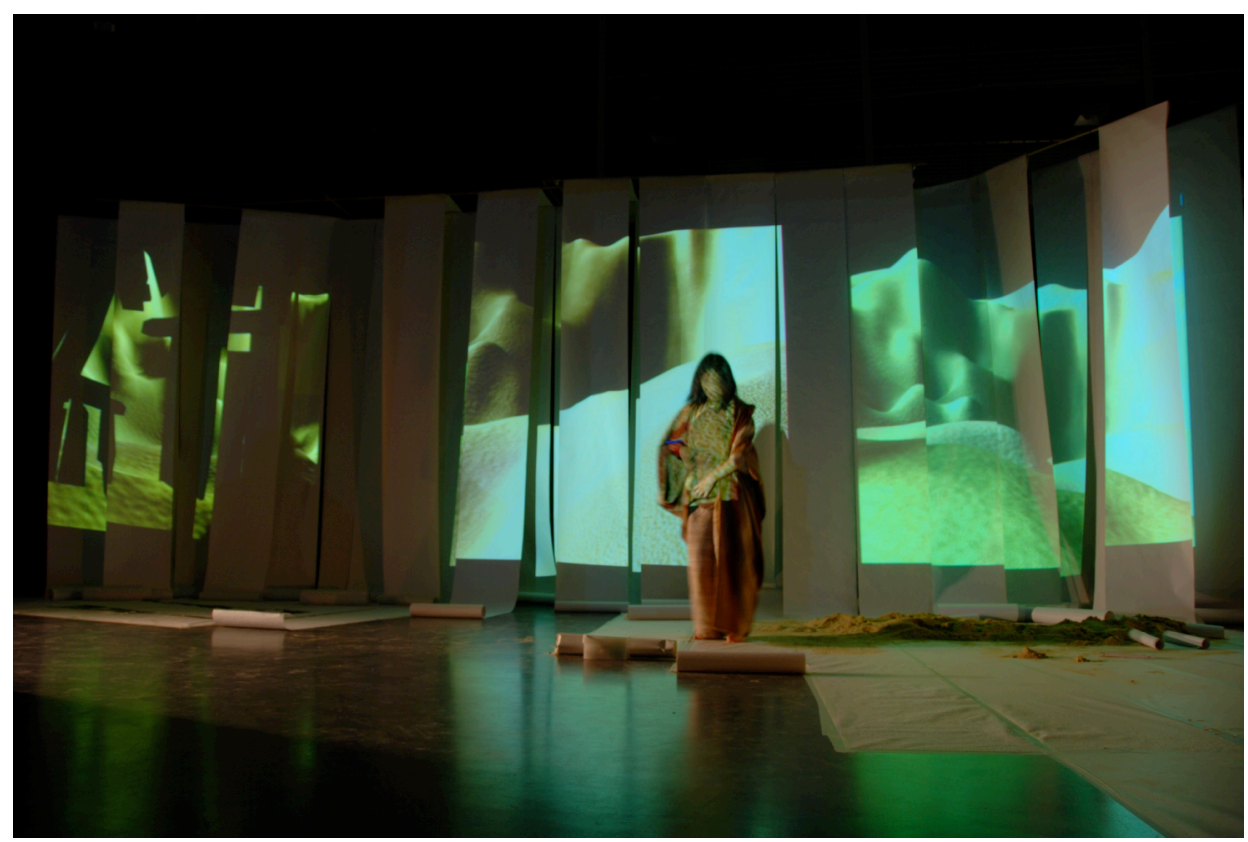

Fig. 6 Katsura Isobe with the morphic SandCoat and fish skin alone in the digital dunes landscape. Photo (C) 2007 Hans Staartjes

Methodologically, our approach to the production of movement in/of the space finally concentrated on the design of the other "wearable" interfaces, integrating an array of sensors into the garments and accessories that allow a combination of (gesture/motion controlled and photoactive) sensing and actuation in the intelligent system to affect an unusual mixture of representational video and abstract digital animation. The interactive prototypes (sleeves, fish skin and coat) were developed through an iterative process involving exchange and dialogue between the dancers and the designer and the rehearsals with choreographer/filmmaker, sensor programmer, 3D animator, composer, scenographer and lighting artist. The completed collection includes the following artifacts:

- DunesWoman: Garment: ([Morphic] SandCoat with Sensors All-in-one, Work suit, Fishskin, with Photocells, Leather Sensor Sleeve, Glove, Belt, Spider Amulet)

- $\quad$ BlueSpirit: (All-in-One [Liquid] Play suit, Black Jersey Sensor Sleeve, 2 pairs of Gloves, Headscarf, Sandpocket)

- $\quad$ FolkWoman: Dress (All-in-One [Rhythm] Sandsuit, Obi, Headscarf, Beetle Amulet, Silk Ties for Hands)

- $\quad$ BeetleBody: Dress (Leather Gilet/Shell, Mask, Sensor Sleeve, Headscarf, Long Gloves, Leg Garters, Leg Warmers, Leggings) 
- $\quad$ EvilVillage: Dress (RecycleDress with Leather Hood, Headscarf, Leg Warmers, Beetle Amulet)

- $\quad$ CaptiveMan: Royal Coat (Coat, with White Shredded Garment)

After testing different types of sensors (touch, bend, orient, heat, magnetic, etc.), the Teshigahara range now incorporates four interactive types: the sleeves and coat integrate accelerometers and the fish skin houses four photocells (light sensors). For the integrated sensor design on the garments, we use an off-the-shelf system (I-CubeX from Infusion Systems). Transmission of data is via Bluetooth with the Wi-microDig (v6.00/6.1), a thumb-sized, easily configurable hardware device that encodes up to 8 analog sensor signals to MIDI compatible messages with high resolution and transmits these messages wirelessly to a computer in real-time for analysis and control purposes. During the programming of the interactive software patches (Isadora and PD), we noted that the I-Cube Editor software is less reliable when there are many simultaneous incoming data streams. Thus we carefully selected the streams for particular scenes of the performance, giving the dancers more time to familiarize themselves with the brittle ways in which the accelerometers inside the cloth responded to directional commands, sensing acceleration (or deceleration) and inclination (tilt, i.e. acceleration due to gravitation). (Fig. 7, 8 and 9)
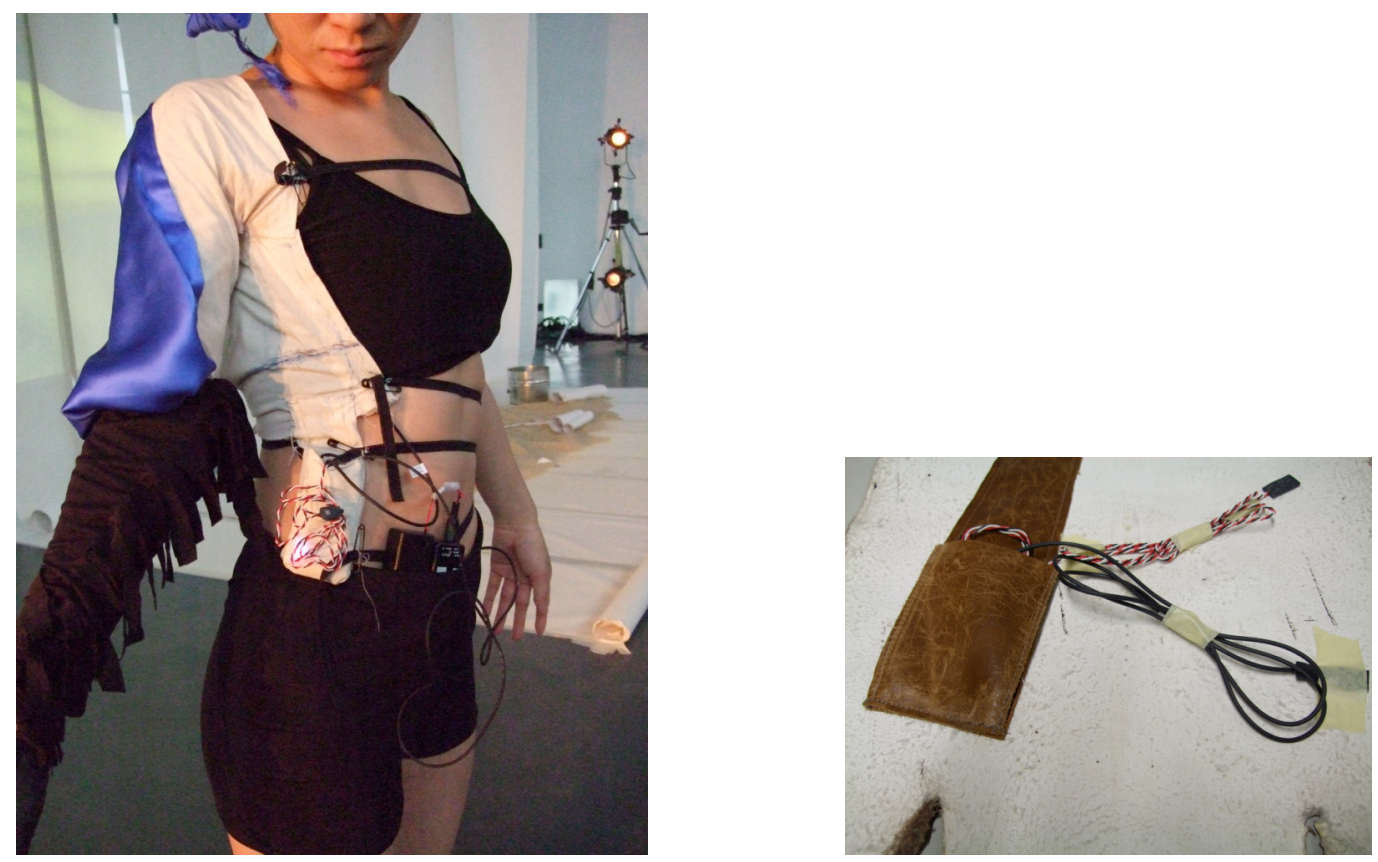

Fig. 7 Test rehearsal with Helenna Ren wearing sensor sleeve and transmitter. Photo (C) 2007 Michèle Danjoux. Fig. 8 Sensor integration into fish skin garment. Photo (C) 2007 DAP-Lab/Matt Bunn. 


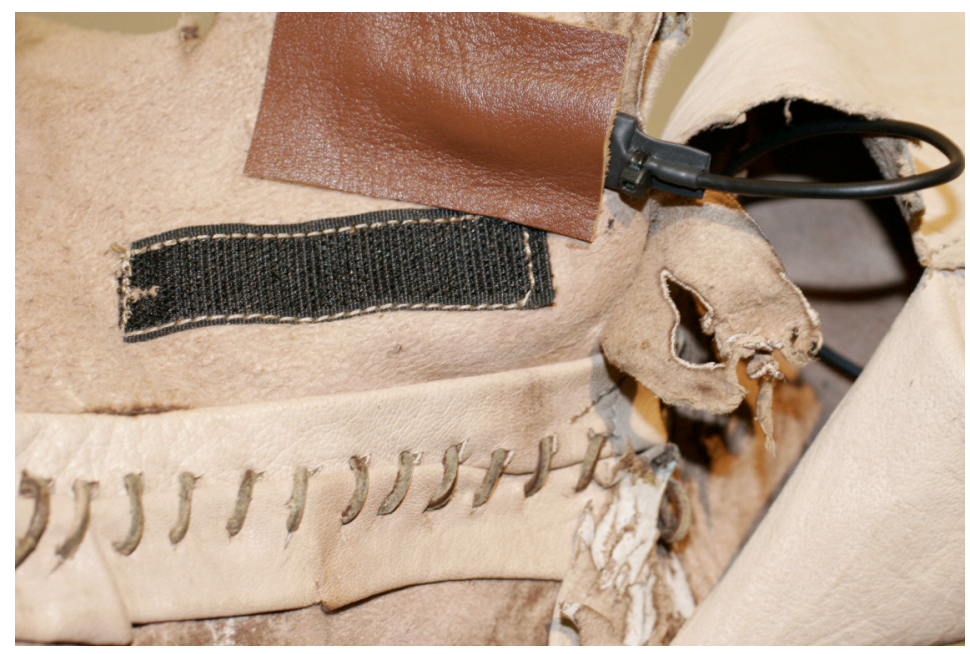

Fig. 9 Pocket for transmitter integrated into fish skin garment. C) 2007 DAP-Lab/Matt Bunn

Over time it becomes apparent that there is an entire codex of (architectural) elements referring to the functional and expressive directional commands of gesture (tilting, rotating right or left, jolting upward, motioning downward, accelerating along the $\mathrm{x}$ axis, decelerating along the $y$-axis, etc). This motional grammar reminds us of the importance of the role of movement in the generation of space as such (motor space, visual space), and current neurophysiological research on the complementarity of sensory information adds a dimension that will be very valuable for future investigation into sensoriality. For example, when we apply sensor technologies to the skin or the clothes, we might augment or interfere with the visual, kinaesthetic (vestibular and proprioceptive) and tactile modalities through which the nervous system senses the external world and enables bodily orientation and cognitive processing. The dancers are not asked to work with sensory substitution, for example replacing visual with tactile information (cf. Lestienne 2008), but to assimilate complex motor activity into a quasi-virtual 3-D environment - the projected image spaces which their movement stimulates.

\section{Embodiment and environment}

The wearer of the sensortized garment thus performs passageways from the inside to the outside within the responsive environment, learning to play with the fabric as a kind of a membrane between the real and the projective, between herself and her 
other, with "control" shifting from fabric to dancer and back. The "wearable" prototypes can respond to micro-movement or larger dynamic actions, and the "thresholds" can be understood architecturally, physically, mentally, as well as technically of course (regarding the data values generated for the software and calibrated for the interactive patches). The performance manifests the transitions between the inside (biological/emotional) and the outside (gesture and movement with the smart materials/garment) which affect the visibly and audibly revealed (screen and sonic architectures).

There are ideally no distinct boundaries between inside and outside: the transition should be fluid from the sensory trigger; the moment of thought and gesture to the movement/shift of images on screen. In the case of the DunesWoman garment, sand tones and rhythmic, organic textures unite the body of the woman with the projected sand. A shape shifting movement sensitive coat (integrating 3 accelerometers) is worn over a rustic silk and sandblasted work suit. Between the work suit and the coat lies a second interactive layer, a protective shield: a fish skin housing 4 light sensors for control of audio and visual output.

In Scene 1 ("Life in the Sand"), we see Isobe working with the coat's sculptural forms and abstract shapes, the "sand woman" on the boundaries between the self and the environment, exploring her garment as the thin membrane. Experimenting with all the characteristics of camouflage in the SandCoat, the woman becomes the camoufleur. With her protective layer of coloration, she can conceal, distort, deceive and even advertise (for the purposes of attracting the man) through the use of disruptive pattern (use of light/dark/variable tone) and "dazzle" techniques (bold contrasts), thus merging the moving object-body with the constantly shifting background of projected sand dunes, which can be said to have attractor states as well since the digital motion implies agent properties. Here the form and colour of the coat are selected to serve the purpose of concealment and disguise, the design constructing an artificial covering and makeshift shroud of sand grains, colours, tones and textures. Wearing this coat, the woman literally adapts to the environment and her ability to employ the tools of the natural world to ensure her survival. (Fig.10) 


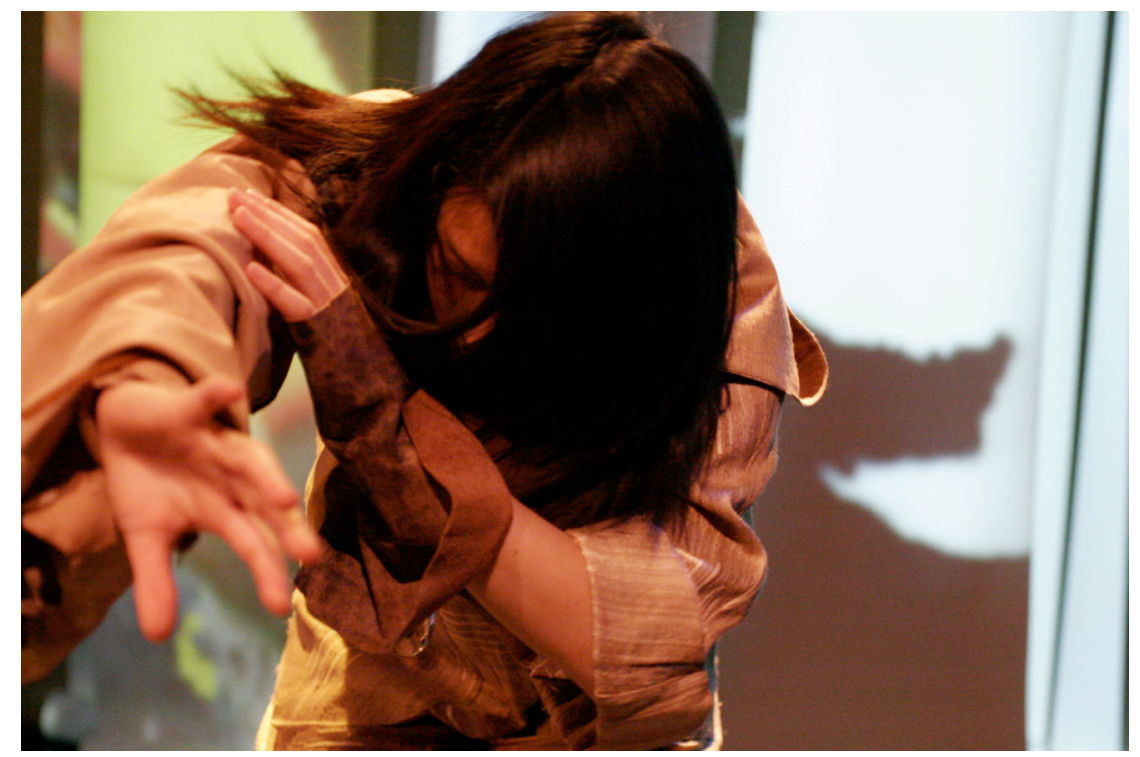

Fig. 10 Katsura Isobe dancing with Leather Sensor Sleeve and Glove, in Scene 1 of Suna no Onna. Photo (C) 2007 Michèle Danjoux

Another example is Scene 7 with BeetleBody, a composition in blue. Unexpected shapes combine in a surreal and fantasy mix of poetic lines. The understated, sensual and liquid form of a hooded silk satin playsuit with luxurious proportions and drawstrings is worn beneath a soft laser cut lamb nappa "beetle shell” belted gilet. Close to the skin is an interactive "fantasy" and insect-like sleeve housing sensor, transmitter and battery pack giving the dancer editing power over her cinematic world. The spirit has a "magical" sensor for the element of play. Infused in both
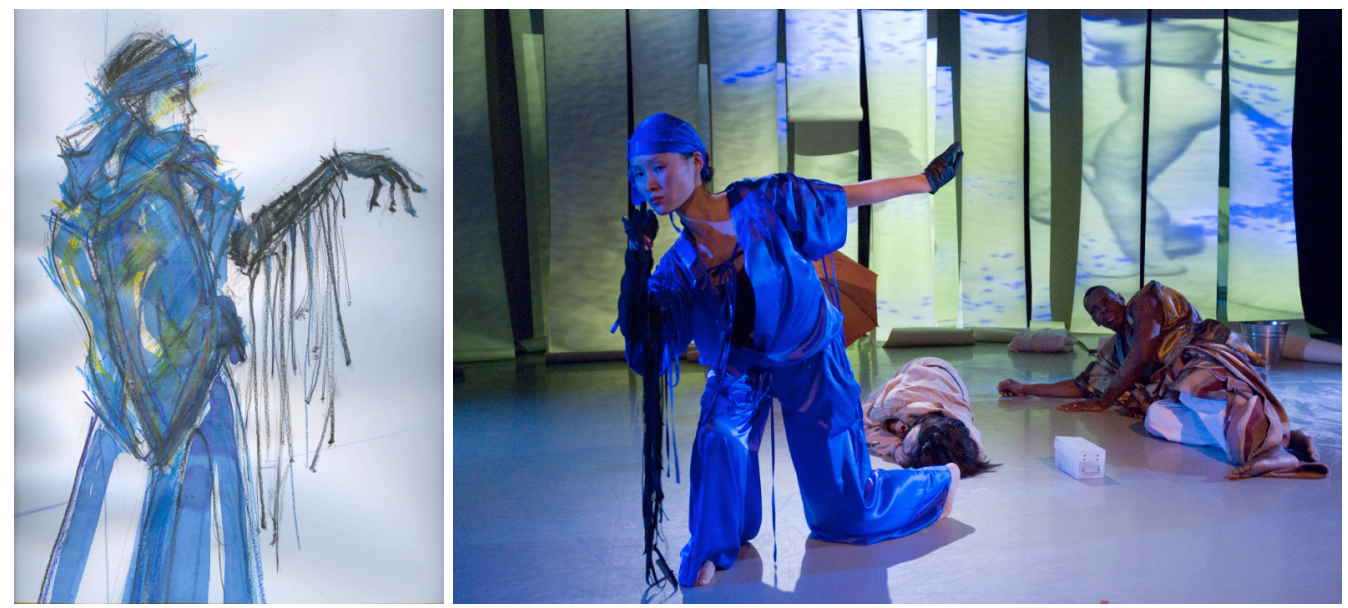

Fig. 11 BeetleBody Garment character, laser cut blue leather gilet and sensor sleeve (illustration) for interactive beetle dance. (C) 2007 Michèle Danjoux Fig. 12 Helenna Ren in the "Spirit Dance" scene, with Katsura Isobe and Olu Taiwo. (C) 2007 Hans Staartjes 
outfits are many shades of Eastern cutting philosophies with inspiration taken from Suna no Onna (both novel and film), Samurai Warriors, beetles, Cosplay and a touch of martial arts. These two pieces (worn by two separate dancers) shift us between the two worlds of the woman, her existential self and her fantasy self, exploring the physical and psychological aspects of wearing. (Fig.11 and 12)

Scence 5 ("Eros") features the fish skin housing the four light sensors which are sensitive to light or temperature. Working with intensity of light-energy, frequency and wavelength we allow the data generated from light directed (by Olu Taiwo) onto the fish skin (worn by Isobe) to take the viewer in and out of a series of black and white stills projected on screen in the love scene. The sequence of stills show the man and the woman making love, naked, hot, sweaty and covered in sand grains (in the humid environment of the dunes). This intimate remotely activated love scene, with the tenderness and vulnerability of the unclothed bodies, intensifies our presumptions about the still clothed bodies and their physical interfaciality (the scene on stage is darkly lit and obscure), as Taiwo's careful gestures seem both tangible and intangible. They heighten the suspenseful oscillation that always occurs between live performance/projected image, the real and the imaginary "chasing after each other," as Deleuze remarked about the crystal-image (Deleuze 1989, p. 127). The integration of sensors once again was carefully mapped onto the movement knowledge generated by the dancers' bodies operating in the responsive environment. (Fig.13)

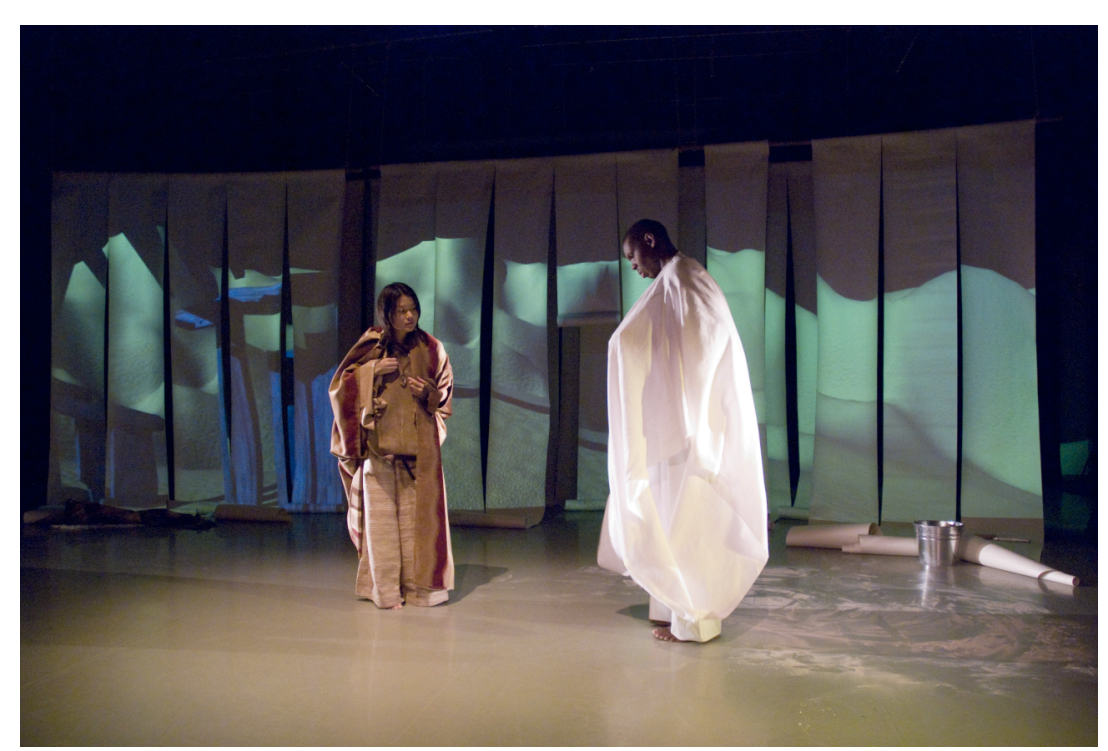

Fig.13 Katsura Isobe and Olu Taiwo inside the projected digital dunes environment, as they prepare for the love scene in Suna no Onna. (C) 2007 Hans Staartjes 


\section{Conclusion: digital anime and post-choreography}

The interactive levels or qualities in the different scenes vary depending on the sensortized garment forms, their textures and weights, and the dramaturgical choices for performer techniques producing material transformations of the digital projections or determining the flow rate of the film stills/still films and micro-loops. In our design vision, the phenomenological embodiment and sensory experience of the performers are coupled with the digital environment which behaves like a living organism: it breathes and moves with the performers who wear the Teshigahara garments.

Since the exhibition of the work is presentational (in a theatre or gallery), audience observation and kinaesthetic empathy are inevitably drawn to the performer-image relationship, to the spatialization of the temporal media and the "logic" of connection. In conventional interactivity, a direct or 1:1 mapping between input and graphic or sonic feedback/representation is often considered desirable, as such transparency allows for mimetic recognition of cause and effect which is pleasurable and (narratively) reassuring. The literal mapping also determines the first-person POV in game design for 3-D environments based on physical modeling, collision detection, and three-dimensional movement. It will become increasingly interesting to ponder "narrative" or "not-narrative" functions and cues of avatar choreographies in 3-D virtual environment, for which the player maintains a corporeal presence and "plays" throughout the game with her body, involving cognitive and sensorimotor processes active in any engagement of spaces that can be seen, heard, felt, and intuited with the bodily intelligence. Instead of a wrist/hand interaction with the interface, whole-body engagement opens up more provocative design challenges for the proprioceptive, cognitive, and physical processing of temporal and spatial experience - as it is fundamental for dance - in 3-D performance interfaces (Birringer 2008).

Yet, the notion of "wearing the film" was first used by Jane M. Gaines in her analysis of specific film dresses that draw attention to the elaborate design in excess of narrative or diegetic function (Gaines 2000). In our case, rather than only (re)appearing as an iconic garment in the virtual space (as with ScreenDress), the SensorGarments allow the dancers to animate occurrences in the virtual dunes. The 
woman protagonist in Suna no Onna appears almost literally fused with the sand habitat; she wears the space as if continually affecting the flow and motion of the realtime filmscape. (cf. Fig. 14) But this analog relationship is deceptive, since there are always differentials in the body/code dynamic, and the non-literal mappings in Suna no Onna give the data flow and internal processing of the digital system a certain autonomy which provides disorienting, not-narrative and unpredetermined emergences. In particular, the images projected on the hanging scrolls also tend towards a diagrammatic, anime-like flatness typical of the Japanese form of digital animation, thus interfering with Western photographic and cinematic realism. Similarly, the very subtle wind and breathing sounds, silences, and sonic disjunctions are duplicitous and can be abrasive, making the affective perturbations between performer and landscape more difficult to parse.

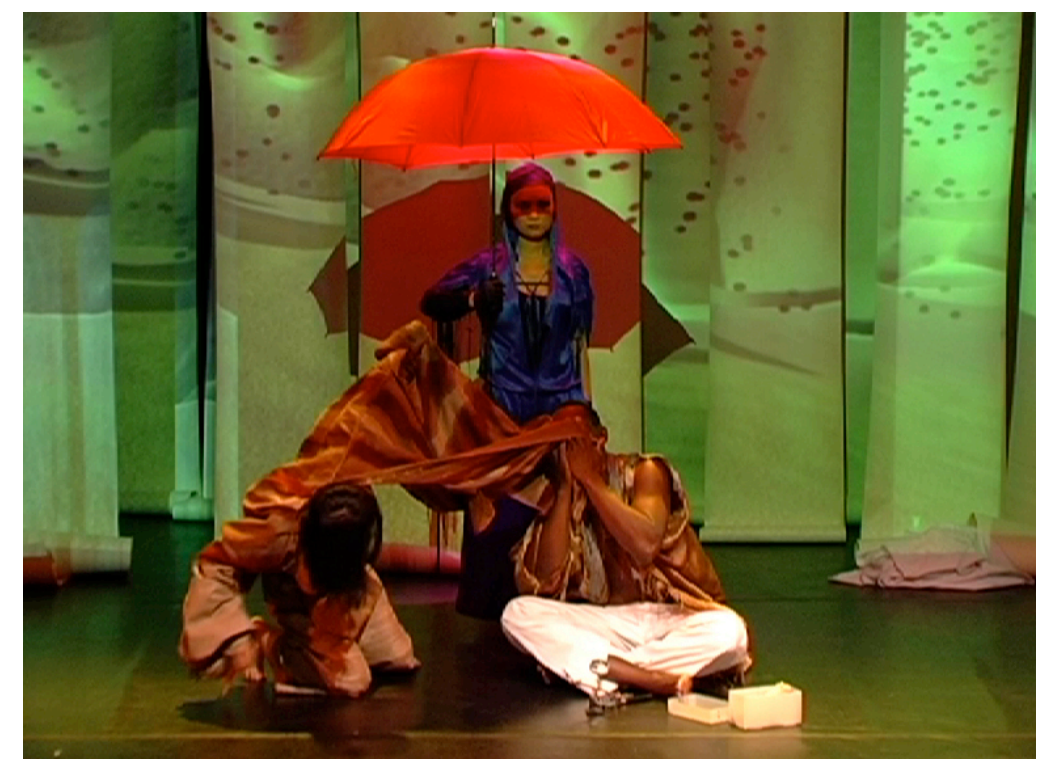

Fig. 14 Digital rain scene, with Katsura Isobe and Olu Taiwo (seated), and Helenna Ren (with umbrella). (C) 2007 Hans Staartjes

The wearing of the shifting, moveable, moldable kimono reveals a world of images. The frames that make up this world float, tremble, hover, fall static, and then shiver and move again - "frames" here referring to digital video/animation frames as film stills/still films on a micro or infra-level. These motions of floating forms, sometimes congealing to a recognizable Gestalt, sometimes flowing as graphic and geometric abstractions, anamorphoses, glitches, distortions of space, must bear on the wearer and the fantasized inside-outside relations. When digital insects appear to crawl out of 
the sand and move about, when carnal and entomological projections are hovering between the phenomenal and the techno-genetic, strange colors and slowed-down animations often create an unnerving and paradoxical stillness, the protagonists trapped in a suspended landscape.

Initially, we thought of the dancer as the sensorimotor actor of the movement-images and surface media, but as the work progressed, the question of control, in the sense of cause-and-effect, became unanswerable, as the programming of the interfaces allows the sensortized garments to move the frames (forward, backward, fast forward, slow down, freeze-frame, jitter, etc) within algorithmic parameters that also allow autonomy to machinic interpolations to generate motion "masks" and corruptions that act as filters for the images.(Fig.15)

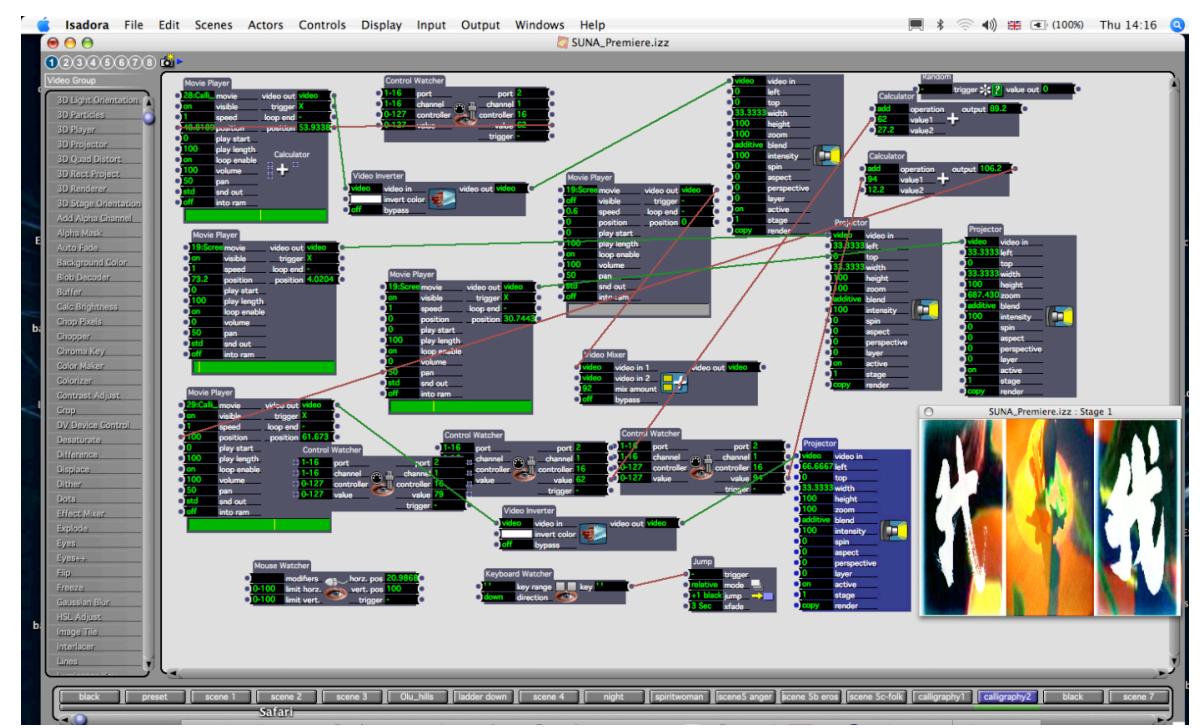

Fig.15 Interactive programming patch (Isadora) for Scene 13 of Suna no Onna. Videostill @ 2008 J. Birringer

Dancer and system interfere with each other on the opaque inframedia level, the numerical substrate where microactivities of fluctuations in data and/or voltage subtend the digital anime (Birringer/deLahunta 2002). The audience does not see the sensors and cannot know the wearable "effects," which we consider a valid option for further artistic research into fashion performance and digital interface design that access sensing technologically from the outside in, through tracking of kinematic data. Whether the audience can infer the somatosensory experience, from outside in and inside out, will depend on their empathetic experience of the coupling in the 
performed, embodied environment which is full of multisensory stimulation. As yet we have not developed an installation concept that might incorporate audiences as interactors invited to learn the performer techniques and aesthetic processes of composition; there are obvious limits to achieving interactant knowledge of dynamic properties in a digital narrative environment through a quick fix.

Our rehearsals with the SensorDress have shown that wearable performance subjectively requires a deep practice and immense dexterity: the performer of a garment-character is responsible for the proprioceptional processing, her physical and affective relationship to garment and animated world, and to create a performance means learning to play the interface. The emotional relationship and expressional exchange between dancer and world, in turn, are affected by the programmable as well as unpredictable behaviors of the worn. In this sense, we speak of the postchoreographic in our work (Birringer 2008c). Although the notion of choreography will not disappear in the context of $21^{\text {st }}$ Century digital performance and virtual art, it undergoes a re-evaluation in terms of how bodily movement produces data or how a performer or immersant engages with an interfacial design environment which is networked and open to contingent, emergent states. These states evolve from the system behavior as a whole, from the digital body-environment interaction.

\section{Acknowledgements}

The "Design in Motion" project and the Suna no Onna dance environment were directed by Johannes Birringer, with fashion design and art direction by Michèle Danjoux, and performed by Katsura Isobe, Helenna Ren and Olu Taiwo; music composition and live processing by Oded Ben-Tal; sensor programming by Paul Verity Smith; digital animations by Doros Polydorou and Maria Wiener (with additional motion graphics by Jonathan Hamilton); scenography by Hsueh-Pei Wang, and lighting design by Miguel Alonso. Produced by Dans Sans Joux (http://www.danssansjoux.org). The research was supported by DAP-Lab/Brunel University Center for Contemporary and Digital Performance, Nottingham Trent 
University School of Art and Design, Interaktionslabor Göttelborn, and the Leverhulme Trust.

\section{References}

Abe, K. (2006) The Woman in the Dunes, trans. Dale Saunders. Penguin, London. Akiyama, K., Hayashi, H., Obari, M., Takayama, F., Goda, H. (1987), Baburugamu kurairshisu (original video animation, Japan).

Baurley, S. (2005) "Interaction design in smart textiles clothing and applications", in Xiaoming Tao (ed.) Wearable Electronics and Photonics. Woodhead Publishers, Cambridge, pp.223-43.

Berzowska, J. (2005) “Memory Rich Clothing”: http://www.berzowska.com

Birringer, J. (2008) Performance, Technology, and Science. PAJ Publications, New York.

Birringer, J. (2008b), "passage: a review," published online:

http://people.brunel.ac.uk/dap/passage.pdf

Birringer, J. (2008c) “After Choreography,” Performance Research vol. 13, no.1, pp. $118-22$

Birringer, J. and Danjoux, M. (2006) "The Emergent Dress: Transformation and Intimacy in Streaming Media and Fashion Performance", Performance Research, vol. 11 , no. 4 , pp. $41-52$.

Birringer, J. and deLahunta, S. (2002) 'New Performance Tools: Technologies/ Interactive Systems," http://minuet.dance.ohiostate.edu/ birringer2//dance_and_technology/ttreport.html

Bokowiec, J. and Bokowiec, M. (2006) "Kinaesonics: The intertwining relationship of body and sound", Contemporary Music Review, vol. 25, no. 1-2, pp. 47-57.

Chalayan, H. (2007) Flip Through, Spring collection 2007; Video Dress, Fall collection 2007: http://www.husseinchalayan.com

Cochrane, L. (2008) “News”, i-D magazine, no.289 (July), pp. 36.

Cute Circuit (2008) http://www.cutecircuit.com

Deleuze, G. (1989) Cinema 2: The Time-Image, trans. Hugh Tomlinson and Robert Galeta, University of Minnesota Press, Minneapolis. 
Franklin, D (2006-08) Micro'be' Fermented Fashion:

http://www.symbiotica.uwa.edu.au

Gaines, J. M. (2000) “On Wearing the Film”, in Fashion Cultures, eds S. Bruzzi and P. C. Gibson. Routledge, London and New York, pp. 159-177.

Gonsalves, T. (2004) Medulla Intimata: http://www.tinagonsalves.com/

Hahn, T. (2009) "Blanche Neige,” ballettanz, vol. 1/09, pp. 12-17.

Hanra, H. (2008) "Together We Make a Great Team," i-D magazine, no.289 (July), pp. $150-61$.

Hansen, L.K. and Kozel, S. (2007) "Embodied Imagination: a hybrid method of designing for intimacy", Digital Creativity, vol. 18, no. 4, pp. 207-20.

Hansen, Mark B.N. (2004) New Philosophy for New Media. The MIT Press, Cambridge, Massachusetts.

Harris, J (2002-03), Potential Beauty: http://www.janeharris.org/page14/page14.htm

i-D magazine (2008), “The Stepping Stone Issue: Learn From The Past, Live In The Present, Look To The Future," no. 289 (July).

Jones, K (2008) “Innovate never Imitate,” i-D magazine, no.289 (July), pp. 122-29.

Kondition pluriel (2008), passage, choreographic installation, presented at CYNETart 2008 festival, Dresden/Hellerau.

Kozel, S. (2008) Closer: performance, technologies, phenomenology. The MIT Press, Cambridge, Massachussetts.

Layne, B. (2006) “Jacket Antics”. Studio subTela: http://subtela.hexagram.ca

Lestienne, F. (2008) "Les sciences du mouvement: art \& handicap," Bains numériques, vol. 2, pp. 81-87.

Maubrey, B. (2008) Audiogruppe: http://home.snafu.de/maubrey/

Munster, A. (2006) Materializing New Media: Embodiment in Information Aesthetics. Dartmouth College Press, Hanover.

Schiphorst, T. (2006) "Breath, skin and clothing: Using wearable technologies as an interface into ourselves", International Journal of Performance Arts and Digital Media, vol. 2, no. 2, pp. 171-86.

Seymour, S. (2008 Fashionable Technology. Springer-Verlag, Wien.

Storey, H. and Ryan, T. (2008) http://www.showstudio.com/wonderland 
Suna no Onna (2007) dance environment created by DAP-Lab/Dans Sans Joux, Laban Centre, 8 December, 2007. Further performances were at Watermans Art Center, March 2008. Film excerpts are available at: http://people.brunel.ac.uk/dap/onna_preview.html

Tanecni zóna (2006) Tanec a Móda/Dance and Fashion, vol. 10, winter issue.

Tedr (2005) performance created by DAP-Lab and ASU, linking Nottingham (UK) and Tempe (Arizona) in a series of telepresence interactions involving dancers and garment design.

Wallace, K. (2008) http://www.kerriwallace.com

Wehrli, P. (2008), camera orfeo, an auto-choreographic composition, first presented at Radialsystem Berlin, and subsequently at CYNETart 2008 festival, Dresden/Hellerau. http://www.aether1.org/

Yamamoto, Y. (2008), “Coming Soon" collection, Fall-Winter 2008. http://uk.youtube.com/watch?v=DSObHV1h37g 